\title{
Mediación para prevenir los factores de riesgo psicosocial
}

\author{
Mediation for the prevention of psychosocial risk factors
}

\section{Resumen}

El presente artículo tiene como objetivo principal mostrar al lector la realidad de los factores de riesgo psicosocial. La globalización, la tecnología y la economía son elementos importantes en la actualidad para el mundo empresarial, pero esto no quiere decir que existan mejoras en la prevención de los riesgos psicosociales por parte de las empresas incluso países, los datos muestran la gravedad de la situación con respecto a los accidentes y enfermedades labores que inciden directamente con la calidad de vida de la clase trabajadora, además la psicología organizacional juega un papel importante en la prevención y protección de los empleados, disciplinas como la seguridad e higiene laboral ayudan a entender cómo se deberían prevenir los riesgos dentro de una organización no obstante, existen mecanismos como los Métodos de Solución de Conflictos (MSC) que contribuirán a solucionar conflictos dentro de las organizaciones y evitar que se den los riesgos psicosociales, de esta manera se abordará específicamente la mediación, el cual es un método avalado científicamente que tiene como objetivo resolver cualquier tipo de conflicto entre empleado y empleador, para así mejorar las condiciones de trabajo y la calidad de vida de los trabajadores.

Palabras clave: Factores De Riesgo Psicosocial, Mediación, Psicología, Seguridad Laboral, Higiene Laboral. 


\begin{abstract}
The main objective of this article is to show the reader the reality of psychosocial risk factors. Globalization, technology and economy are currently important elements for the business world, but this does not mean that there are improvements in the prevention of psychosocial risks by companies even countries, the data show the seriousness of the situation with respect to accidents and occupational diseases that directly affect the quality of life of the working class, also organizational psychology plays an important role in the prevention and protection of employees, Disciplines such as occupational health and safety help to understand how to prevent risks within an organization, however, there are mechanisms such as Methods of Conflict Resolution (MSC) that will help to resolve conflicts within organizations and prevent psychosocial risks, in this way we will specifically address mediation, which is a scientifically supported method that aims to resolve any type of conflict between employee and employer, in order to improve working conditions and quality of life of workers.
\end{abstract}

Key words: Psychosocial Risk Factors, Mediation, Psychology, Occupational Safety, Occupational Hygiene.

\section{EL TRABAJO COMO RIESGO LABORAL}

En el transcurso del tiempo se ha demostrado que las empresas están sometidas a cambios constantes como la tecnología, economía, globalización y la cultura organizacional, (Rivas-Castillo, Rodríguez-Burgos \& Miranda-Medina, 2021). Esto a su vez genera incertidumbre en lo que concierne al ambiente y condiciones de trabajo que es donde se manifiestan los accidentes y enfermedades laborales, no obstante, la seguridad en el trabajo se considera eje fundamental para garantizar la salud de los trabajadores.

Cabe señalar que el trabajo ayuda a la autorrealización personal sin embargo, también es una fuente de daño a la salud tanto psicológica como física, la clase trabajadora ha estado carente de la protección del empleador, pues a través de la historia ha sido evidente que no ha tenido la protección que se merece, pero el panorama ha cambiado y la aparición de los primeros grupos sindicales que apoyan los derechos humanos y la forma de producción empresarial ha disminuido la frecuencia inhumana de las condiciones de trabajo (Moreno-Jiménez, 2011).

Es importante señalar que dentro de las organizaciones se presentan una serie de eventos y situaciones que de alguna manera intervienen en el desarrollo de las actividades del trabajador. Una organización para (Barnard, 1971 como se citó en Chiavenato, 2007) es un sistema de actividades conscientemente coordinadas entre dos o más personas, el cual es esencial para la existencia de la organización, además esta varía enormemente no sólo por las diferencias individuales sino también por el sistema de recompensas que emplean las organizaciones. 
Sin importar la actividad que sea, aunque algunas empresas generan más riesgos que otras en determinadas actividades, en toda empresa la seguridad deberá empezar por los altos ejecutivos, seguir por los funcionarios importantes y concluir en los trabajadores, y deberán tener presente que es un imperativo legal plasmado en las constituciones de los países. La seguridad debe organizarse de manera que responda a la realidad particular de cada empresa, buscando obtener el máximo de seguridad con el mínimo de inversión, siendo que ésta debe ser considerada como más una inversión que un gasto.

La globalización ha transformado no solamente el ámbito social sino también el laboral, lo que supone tener más personal capacitado, especializado y comprometido con su trabajo, precisando que las empresas realicen esfuerzos para posicionarse en los mercados, transfigurando en cierta medida, la forma como se hacen las cosas dentro de la empresa. Estos cambios han permitido adoptar medidas en la seguridad en el trabajo reduciendo determinados riesgos, sin embargo, la automatización de los procesos y los nuevos equipos tecnológicos han dado paso a otros tipos de riesgos (Garrido-Pinzón y Uribe-Rodríguez, 2011).

De este modo el mundo laboral se ha convertido en un fenómeno complejo y cambiante, como observamos en el informe del 2006 de la Conferencia Internacional del Trabajo, en el que se identifican 4 motores del cambio en el mundo del trabajo que son: 1) imperativo del desarrollo 2) la transformación tecnológica 3) la intensificación de la com- petencia a escala mundial y 4) una mayor función de los mercados y una función más reducida del estado; estos cambios además de afectar las economías de los países tendrán consecuencias sobre la población laboral activa (Monte-Gil, 2012).

En las organizaciones suceden eventos que pueden generar daño al trabajador como son los accidentes de trabajo y las enfermedades laborales. Según Murva (1977) un accidente de trabajo es "un suceso eventual debido a contacto o exposiciones de objetos, substancias, personas o animales y que alteran el orden de un proceso normal, implicando generalmente lesión personal, daño material o ambos" (p. 55). Las enfermedades laborales "son los objetos o situaciones ligadas con el trabajo que, al operar de manera continua sobre las personas del trabajador, causan o inducen en este un estado patológico" (Aguirre, 1994, p. 14).

Con respecto a los accidentes de trabajo, su aparición es inmediata al contacto con el objeto, sustancia, persona o animal causando la lesión en el trabajador en el mismo instante, a diferencia de las enfermedades profesionales cuya aparición es gradual y lenta, permaneciendo el mal oculto hasta llegar al estado patológico por el transcurrir del tiempo en sus labores diarias.

Ahora bien, es importante conocer los riesgos biopsicosociales ligados a los accidentes y enfermedades laborales que suceden dentro de la organización y como afectan al bienestar del trabajador, tener claro que en esta problemática existen conceptos relacionados en la búsqueda de la seguridad 
y el bienestar del trabajador. Es por esto que surge la salud ocupacional como área de control de los riesgos organizacionales (Rentería, Fernández, Tenjo y Uribe, 2009).

\section{SALUD OCUPACIONAL Y SEGURIDAD E HIGIENE LABORAL}

Ruiz (1987) comenta que al mismo tiempo que ocurrían cambios acelerados durante la segunda guerra mundial, organizaciones importantes iban emergiendo así, desde 1919 se funda la Organización Mundial del Trabajo (OIT), que busca mejorar las condiciones de los trabajadores, estableciendo la paz basada en la justicia social así mismo, dentro de la Organización de las Naciones Unidas (ONU) se creó una agencia que tenía como función orientar al mundo en materia de salud humana, la Organización Mundial de la Salud (OMS) que tiene como finalidad buscar en el ser humano un estado de bienestar físico, mental y social. Como resultado de esta labor en equipo, la conferencia internacional del trabajo en 1953 formalizó la recomendación número 97 en la que aconseja a los estados miembros de la ONU la conveniencia de adoptar, con carácter obligatorio, el servicio de salud ocupacional en las empresas y con base en el informe del comité mixto de la OIT-OMS de 1978.

De esta manera la (Organización Panamericana de la Salud (OPS) y OMS, 2003) comentan que según la OIT y la OMS, la salud ocupacional es:

la promoción y mantenimiento del mayor grado de bienestar físico, mental y social de los trabajadores en todas las ocupaciones mediante la prevención de las desviaciones de la salud, control de riesgos y la adaptación del trabajo a la gente, y la gente a sus puestos de trabajo. (p. 3)

La salud ocupacional se apoya en dos disciplinas, que son seguridad en el trabajo e higiene laboral que son las encargadas de prevenir los accidentes de trabajo y enfermedades laborales y además ayudan a mantener a los trabajadores fuera de los riesgos inherentes a las actividades que realiza.

En relación a la seguridad en el trabajo Aguirre (1994) señala:

Evidentemente es ventajosa a que sin siniestro en los trabajadores se evitan las graves consecuencias humanas en perjuicio de estos y las graves repercusiones en la producción con motivo de las ausencias por los motivos expresados; la seguridad en el trabajo es también un imperativo legal a partir de las leyes emanadas por los estados. ( $p$. 174)

Lo que busca la seguridad en el trabajo es evitar los riesgos que proceden de las prácticas inseguras o condiciones del medio ambiente en donde se realizan las actividades diarias, ya que se podría provocar consecuencias muy negativas tanto en la pérdida de producción como de vidas humanas, es por esta razón que los Estados han hecho de estas ciencias un pilar importante con lo que se refiere a las leyes que buscan proteger al trabajador. 
La seguridad laboral no es sólo un conjunto de normas escritas, ni los medios de protección, ni los carteles o los colores de señalización, la seguridad es una filosofía operativa de la dirección que debe ser aplicada con la misma firmeza que la gente se pone en buscar una producción, en la calidad y cantidad a precio rentable, teniendo encuentra el peso del factor humano en el proceso productivo.

En este mismo orden de ideas la seguridad y la salud en el trabajo son actividades que se interrelacionan primordialmente con las personas que se encuentran en su puesto de trabajo, como los materiales, maquinarias, medio ambiente y productividad de este modo la seguridad beneficia de forma directa al personal y la empresa, no obstante, sus objetivos básicos son; evitar lesiones y muertes por accidentes, reducción de los costos operativos de la producción, mejorar la imagen de la empresa.

Para Cortés (2015) por seguridad del trabajo se entiende "la técnica no médica de prevención cuya finalidad se centra en la lucha contra los accidentes de trabajo, evitando y controlando sus consecuencias" (p.78). El mismo autor comenta el objetivo primordial de la seguridad en el trabajo que es la lucha contra los accidentes de trabajo, lo que permite distinguir a la seguridad de otras técnicas no médicas de prevención, como la higiene del trabajo.

Poza (1990) indica que:

La higiene industrial se refiere principalmente al control de las perturba- ciones de la salud, debidas al medio ambiente laboral y se concretan en el estudio de las, a) condiciones que causan las enfermedades profesionales legalmente reconocidas, b) cualquier otra clase de condiciones en el puesto de trabajo que no produzcan enfermedades, si no que puedan influir de algún modo en la salud de los trabajadores, de tal forma, que les haga perder tiempo o eficiencia. (p. 19)

El mismo autor señala que la higiene industrial se dirige, por lo tanto, al conocimiento, evaluación y control de estos factores ambientales químicos, físicos, biológicos, ergonómicos y psicosociales que pueden producir enfermedades, desequilibrar la salud o disminuir el confort.

Según Aguirre (1994), "la higiene del trabajo es la parte de la medicina que tiene por objeto la conservación de la salud y los medios de precaver las enfermedades" (p. 57). En consecuencia, para aplicar la higiene del trabajo se deberá observar, establecer y además vigilar las condiciones que conlleven y ayuden a conservar y mantener un medio de trabajo lo suficientemente sano y de esta manera evitar al máximo enfermedades que, en un momento dado, pueden transformarse en cuadros epidémicos o endémicos.

En este mismo orden de ideas, tal como lo exponen, Ruiz y Pañela (1955) cada una de las dos disciplinas antes nombradas, cumplen con su finalidad por separado, la primera busca evitar los accidentes de trabajo y las consecuencias que sucedan de estos tales como; fracturas, golpes, heridas, 
luxaciones, quemaduras y en el mayor de los casos la muerte y la segunda conservar la forma de precaver las enfermedades en el trabajo y conservar la salud de los trabajadores en sus actividades diarias buscando un equilibrio biopsicosocial del ser humano dentro y fuera de la organización.

Las estadísticas de la OIT (2020) no son muy alentadoras ya que cada día mueren personas por enfermedades y accidentes laborales, en el 2020 fueron 2,78, mientras que los trabajos mortales originan alrededor de 374 millones de lesionados, esto se traduce en un ausentismo laboral promedio de 4 días, además, todos estos eventos afectan el Producto Interno Bruto global (PIB) cada año estimado en un $3,94 \%$ por malas prácticas laborales.

Para la OIT los riesgos en el lugar de trabajo como el ruido, el calor, el polvo, los productos químicos peligrosos, las máquinas inseguras y el estrés psicosocial son causas de enfermedades ocupacionales, además, las condiciones de empleo, la posición y la ocupación en la jerarquía del lugar de trabajo también provocan problemas en la salud.

Así mismo la seguridad y la salud en el trabajo deben ser consideradas y planeadas desde antes de que se materialice físicamente la empresa porque preverá los peligros que la actividad engendre y va a evitar tales riesgos mediante la adopción y aplicación de las medidas adecuadas, para el efecto la seguridad integral en las empresas tiene que contemplar que estas se mantengan exentas de riesgos en materia de accidentes y enfermedades de trabajo, incendios y delitos.
Con respecto a los factores de riesgo psicosocial el Comité Mixto Organización Internacional del Trabajo, OIT - OMS define los factores psicosociales como la interacción entre el trabajo y su medio ambiente, la satisfacción del trabajo y las condiciones de su organización, las capacidades del trabajador, sus necesidades, su cultura y su situación personal fuera del trabajo, todo lo cual, a través de percepciones y experiencias, pueden influir en el rendimiento y trabajo, la satisfacción en el mismo y la salud del trabajador (Díaz, 2011).

Debido a estas variaciones observamos que el perfil de las enfermedades y accidentes laborales ha cambiado y los riesgos psicosociales y sus consecuencias han cobrado protagonismo (Monte-Gil, 2012). El riesgo laboral ha ido evolucionando a lo largo de la historia, en sí mismo el término de riesgo laboral supone un logro ya que durante mucho tiempo ha carecido de sentido. El trabajador no tenía ningún derecho, el trabajo era una obligación y si sufría algún daño era su suerte, el concepto de riesgo laboral aparece implícito o explícito del derecho a la integridad física y a la salud, algo que aparece en la edad media y en el renacimiento; los gremios hacen su primera aparición como sindicatos y suponen la primera defensa formal contra determinadas condiciones laborales y trato a los trabajadores, es así que el riesgo laboral contra la salud aparece definido como las situaciones o conductas que no pueden ser aceptadas por sus nocivas consecuencias para los trabajadores (Moreno-Jiménez, 2011). 
Con respecto a los riesgos psicosociales las formas más comunes de manifestación son el estrés y la violencia laboral (Raffo, Ráez \& Cachay, 2013). Las enfermedades que afectan la salud mental son consideradas en la actualidad un importante problema de salud pública, que no sólo afecta la salud sino también la productividad de millones de personas; uno de los trastornos más comunes dentro de este grupo es la depresión, con una prevalencia de cronicidad entre el 8 y el $15 \%$ a nivel mundial; es así como en el 2013 aproximadamente un millón 868 mil 173 personas sufrieron esta enfermedad y la depresión constituye además la segunda causa de discapacidad en el mundo (Vieco \& Abello, 2014).

Existe una variedad de conceptos con relación a los factores de riesgo psicosocial, de esta manera los autores que investigan esta vertiente laboral exponen el suyo propio, con variadas teorías, y conceptos, enfoques y estudios relacionados con la el fenómeno de estudio que se aborda.

\section{LA TEORÍA DE LOS FACTORES HIGIÉNI- COS Y FACTORES MOTIVACIONALES $Y$ LA TEORÍA DE LA MOTIVACIÓN Y LAS NECESIDADES HUMANAS}

La teoría de los dos factores desarrollada por Herzberg (1987) busca explicar el comportamiento laboral de los individuos. Según esta teoría, existen dos factores los higiénicos y los motivacionales. Los primeros se localizan en el ambiente que rodea al individuo y se refieren a las condiciones en las cuales desempeña su trabajo que no dependen del sujeto ya que la empresa es la que los determina, encontrando entre estas condiciones los salarios, los beneficios sociales, el tipo de jefatura o supervisión que el individuo experimenta, las condiciones físicas de trabajo, la política de la empresa, el clima de relaciones entre la dirección y el individuo, los reglamentos internos entre otros.

Por otro lado, los factores motivacionales o factores intrínsecos están relacionados con el contenido del cargo o con la naturaleza de las tareas que el individuo ejecuta, estas están bajo el control del individuo y comprenden los sentimientos de autorrealización, crecimiento individual y reconocimiento profesional. En otras palabras, la teoría de los dos factores establece que la satisfacción en el cargo es función del contenido del cargo o de las actividades excitantes y estimulantes de éste: son los factores motivacionales; y la insatisfacción en el cargo es función del contexto, es decir, del ambiente, del salario, de la supervisión, de los colegas y del contexto general del cargo.

Maslow (1954) formuló su concepto de jerarquía de necesidades que influyen en el comportamiento humano. Este autor concibió esa jerarquía porque el hombre es una criatura que demuestra sus necesidades en el transcurso de la vida. En la medida en que el hombre las va satisfaciendo, otras más elevadas toman el predominio del comportamiento.

Se identifican cinco niveles y cada uno de ellos se activa solamente cuando la necesidad del nivel inmediatamente inferior está razonablemente satisfecha. 
Donde la mayor parte de las personas en las sociedades con un alto nivel de vida tienen sus necesidades de los tres primeros niveles (fisiológicas, de seguridad y sociales) regularmente satisfechas, sin mucho esfuerzo y sin mucho efecto motivacional, después le siguen las necesidades de estima y autorrealización.

Necesidades fisiológicas: la satisfacción de estas primeras necesidades comprende a los alimentos, temperatura del cuerpo, cuando estas necesidades no son satisfechas por un período largo de tiempo la satisfacción de las otras necesidades pierde importancia por lo que estas dejan de existir.

Necesidades de seguridad: están relacionadas con el mantenimiento de un estado de orden y seguridad. Dentro de estas necesidades se encontrarían las de sentirse seguros, la de tener estabilidad, la de tener orden, la de tener protección y la de dependencia.

Muchas personas dejan suspendidos muchos deseos como el de libertad por mantener la estabilidad y la seguridad.

Muchas veces las necesidades de seguridad pasan a tomar un papel muy importante cuando no son satisfechas de forma adecuada, lo que se ve en la necesidad que tienen muchas personas de prepararse para el futuro y sus circunstancias desconocidas. Necesidades de amor y de pertenencia: se encuentran aquellas orientadas de manera social; la necesidad de una relación íntima con otra persona, la de ser aceptado como miembro de un grupo organizado, la de un ambiente familiar, la de vivir en un vecindario familiar y la necesidad de participar en una acción de grupo trabajando para el bien común con otros.

Necesidades de estima: son aquellas que se encuentran asociadas a la constitución psicológica de las personas. Estas necesidades en dos clases: las que se refieren al amor propio, al respeto a sí mismo, a la estimación propia y la autoevaluación; y las que se refieren a los otros, las necesidades de reputación, condición, éxito social, fama y gloria. Las necesidades de autoestima son generalmente desarrolladas por las personas que poseen una situación económica cómoda, por lo que han podido satisfacer plenamente sus necesidades inferiores. En cuanto a las necesidades de estimación del otro, estas se alcanzan primero que las de estimación propia, pues generalmente la estimación propia depende de la influencia del medio.

Necesidades de desarrollo: las necesidades de autorrealización son únicas y cambiantes, dependiendo del individuo están ligadas con la necesidad de satisfacer la naturaleza individual y con el cumplimiento del potencial de crecimiento. Uno de los medios para satisfacer la necesidad de autorrealización es a través de la actividad laboral o vocacional que uno quiere desempeñar $y$, además, llevarla a cabo del modo deseado. Es importante analizar estas teorías ya que el individuo es un ser de motivaciones que de alguna forma están precedidas por las necesidades, lo que buscan todos los involucrados dentro de una organización es mejorar las condiciones de trabajo y la motiva- 
ción detrás de esto es mejorar la calidad de vida de cada uno de los integrantes de la organización.

Esto tiene relación con lo expresado por Aguirre (1994) quien sostiene, que evidentemente las empresas sea cual fuere su forma de sobrevivir en el mercado, la protección que desarrolle en el trabajador es la clave para fomentar y mantener la buena marcha de las actividades laborales, con el fin de que las personas mantengan la totalidad de sus actitudes dentro de los puestos de trabajo, claro está que generar unas condiciones óptimas en los puestos de trabajo no es responsabilidad de una sola persona ya que todos los involucrados en la organización deben aportar sus experiencias y conocimientos con la finalidad de buscar prácticas fuera de riesgos dentro de la organización.

\section{FACTORES DE RIESGO PSICOSOCIAL, PSICOLOGÍA ORGANIZACIONAL Y ME- DIACIÓN}

El concepto de factores de riesgo psicosocial en el trabajo es reciente y tiene su génesis en el último cuarto del siglo pasado. Una de las primeras referencias oficiales al tema aparece en 1984 con el estudio "Los factores psicosociales en el trabajo: reconocimiento y control", un documento publicado por la OIT, en el que se argumenta que dichos factores son difíciles de entender dado que abarcan muchos aspectos y obedecen a la percepción y experiencia del trabajador (Moreno-Jiménez, 2011).

En el mismo orden de ideas se considera que los factores psicosociales son condicio- nes presentes en situaciones laborales relacionadas con la organización del trabajo, entran variables como el tipo de puesto, la realización de la tarea, y el entorno donde se realiza afectando el desarrollo del trabajo y la salud de las personas trabajadoras, así como los conflictos entre las relaciones de pareja ( Miranda-Medina, Martínez, Salvador, Hernández, Rodríguez y Sosa, 2019) de este modo pueden favorecer o perjudicar la actividad laboral y la calidad de vida laboral de las personas (Monte-Gil, 2012).

Teniendo en cuenta lo anterior, los factores de riesgos psicosocial producen en el trabajador patologías asociadas a la actividad que realizan en las organizaciones. Queda claro que es muy importante determinar si la mediación como estrategia mejora los procesos de comunicación entre empleados y empleadores y si son efectivos para una toma de decisiones correcta y disminuyendo así los efectos negativos en la organización y la salud del trabajador, considerando la normativa laboral para garantizar los derechos de los trabajadores (Martínez-Pérez, Sauceda y Moreno-Rodríguez, 2021).

Del mismo modo, es de gran importancia mantener unas óptimas condiciones ambientales ya que el medio que rodea influirá decisivamente en el desarrollo de las actividades, por lo tanto, unas buenas condiciones ambientales proporcionan una eficiente y segura operación de trabajo, por ejemplo el espacio adecuado para el funcionamiento de las actividades de la empresa como la zona de caldera, el espacio para colocar las herramientas, los pasillos adecuados para circular y además contar con espacios de seguridad. 
Ramírez (2002) explica que, "el ambiente de trabajo es factor esencial en el rendimiento humano; por lo que es necesario que el hombre no trabaje más allá de los límites máximos de su resistencia y en condiciones ambientales adecuadas" (p. 156).

El mismo autor afirma que el medio de trabajo es el resultado de elementos como:

1. Progreso tecnológico: procedimientos nuevos en los sistemas de trabajo, por ejemplo, corte de metales con soplete de plasma.

2. Diversidad de equipo que producen: ruidos, exhalaciones de gas, entre otros.

3. Métodos modernos de organización del trabajo: como el uso de cadenas de fabricación en las que interviene una serie de equipos y sustancias nocivas.

4. Disposición de plantas o distribución: adaptando el elemento físico de la fábrica a las condiciones de las actividades productivas.

Según Aguirre (1994), "el ambiente, propiamente definido, será el conjunto de factores y elementos que estén presentes en el área de trabajo y en el momento preciso en el cual se está desarrollando una actividad" (p. 27). Por estas razones es importante mantener un ambiente apropiado dentro de cualquier empresa, dado que el hombre es un "animal de costumbres" el medio que lo rodea influirá decisivamente en el desarrollo de sus actividades, por lo que un buen ambiente de trabajo proporcionara una eficiente y segura operación de trabajo. Los accidentes y enfermedades en el trabajo no deben considerarse algo natural o inevitable, sino que normalmente son consecuencia de unas condiciones de trabajo inadecuadas.
Los riesgos psicosociales asociados al trabajo son el resultado de aspectos organizacionales, ambientales o personales que, cuando se enlazan con el trabajo, conllevan a riesgos que bajo determinadas condiciones de magnitud y tiempo de exposición afectan la salud de los colaboradores, en sus esferas emocionales, cognoscitivas, comportamentales y fisiológicas, (Charria, Sarsosa, Kewy y Arenas, 2011; Rentería, Fernández, Tenjo y Uribe et al, 2009) manifestándose con mayor frecuencia mediante la afectación de los sistemas cardiovascular, músculo-esquelético, endócrino, gastrointestinal, así como su incidencia en la diabetes tipo II, en los desórdenes del sueño, las disrupciones en la relación trabajo-familia y en trastornos como depresión, ansiedad y alteraciones psiquiátricas menores (Vieco \& Abello, 2014).

De este modo la evolución de la seguridad y salud ocupacional es de vital importancia para la mejora de estas condiciones de trabajo; se hace énfasis en los simples programas de entrenamiento, pasando por las auditorías, realización de análisis de riesgos con base en técnicas avanzadas como HAZOP y FMEA, y en el sistema de gestión OSHAS como último requisito en este proceso (Raffo, Ráez y Cachy et al., 2013).

Cabe señalar que la psicología juega un papel importante en los factores de riesgo psicosocial, es por ello que a lo largo de los años ha centrado sus esfuerzos en buscar una solución al mundo patológico, anteriormente el trabajador era visto como un sujeto pasivo que sólo reaccionaba a estímulos, sin embargo, desde la psicología aplicada, se 
ha puesto énfasis en la cura del sufrimiento, de este modo la psicología se sustenta como disciplina científica y práctica profesional en la medida que es evocada al bienestar humano. De esta manera la psicología de las organizaciones ha tenido un acercamiento a mejorar las condiciones de vida de las personas en su rol de empleado, es por eso que en lugar de representar un costo, la salud y el bienestar se consideran una inversión (Polo-Vargas, Palacio, De Castro, Mebarak y Velilla 2013).

La psicología organizacional tiene como objetivo principal buscar el bienestar biopsicosocial de los trabajadores, ya que las personas pasan buena parte de sus vidas en las organizaciones. Por esta razón prevenir los riesgos psicosociales en el trabajo es fundamental para alcanzar el bienestar y generar condiciones de trabajo estables para cada uno de los trabajadores, contribuyendo a fomentar estrategias empresariales para alcanzar el éxito integral de la organización.

De esta manera se agradece a todas las personas involucradas científicos, psicólogos, mediadores, árbitros y todos lo que aportaron su conocimiento para resguardar al trabajador de tantas contingencias a las que se encuentran sometidos y no permitir que tengan la incertidumbre de que algo va a pasar, bien está el curar, pero es más efectivo el prevenir los riesgos psicosociales en los lugares de trabajo.

Atendiendo a esta problemática, han surgido mecanismos que permiten la prevención de conflictos que se dan dentro de las organizaciones. Con respecto a los Métodos
Alternos de Solución de Conflictos (MASC) Gorjón (2016) así como Durand y Rodríguez-Burgos (2015) indican que están en una etapa instrumental y de cientificidad, considerados como una solución viable y productora de innovaciones asertivas responsables que atiende a los intereses de las partes y conciliar con los intereses superiores de la sociedad en general como modelo de producción del estado. En el mismo orden de ideas Gorjón y Pesqueira, (2015) señalan que los MASC al ser métodos alternativos su operación puede darse dentro y fuera del proceso judicial, ya sea de forma paralela o independiente o pude darse sin que exista el proceso judicial que haya sido motivado por las partes mismas y no por un juez, en todos los casos con la misma validez, además los MASC son un procedimiento universal e internacional con secuencia lógica y técnicas que son parte de un método.

Uno de los MASC más utilizados es la mediación ya que es un componente esencial en la forma de resolver algún tipo de conflicto que se presente dentro de las organizaciones. Bajo este concepto, con la mediación transformativa se obtienen resultados benéficos en cuanto a los procesos psicosociales (Sauceda \& Martínez, 2018). Las condiciones que se encuentran dentro de la empresa obedecen a la naturaleza propia de dicha organización, teniendo claro esto, se puede determinar que el empleador no tiene la motivación de mejorar las condiciones de trabajo de sus empleados ya que estos le generan gastos y tiempo que son las variables que más tienen en cuenta los gerentes a la hora de restructurar las condiciones en el área de trabajo, es por esta razón que es 
necesario que exista una mediación entre el empleado y empleador.

Así mismo tal como lo comenta Bonafé-Schmitt (2006) la mediación más que una técnica eficaz de resolución de conflictos, es un proceso educativo para los participantes, generando solidaridad en las diferentes comunidades y los miembros que la integran, sin dejar de mencionar algunos programas restaurativos, tales como, los encuentros restaurativos, los círculos de paz y las conferencias familiares (Martínez \& Gorjón, 2018).

En el mismo orden de ideas De Armas (2003) comenta que la mediación es un proceso en el que las dos partes acuden voluntariamente y una tercera persona imparcial llamada mediadora la cual busca un acuerdo satisfactorio en este proceso extrajudicial. Se considera un proceso creativo ya que busca que las partes satisfagan sus necesidades y no restringirse a lo que la ley dictamina, además la solución no es impuesta por árbitros o jueces sino más bien por las partes.

De lo expuesto anteriormente lo que se busca con la mediación es tratar de conciliar con las partes involucradas para que no existan ni ganadores ni perdedores más bien generar espacios para mejorar la convivencia, ambiente de trabajo, clima organizacional con la finalidad de prevenir los riesgos psicosociales y estos no afecten al trabajador y causar una enfermedad laboral $\mathrm{o}$ un accidente de trabajo. Además, la psicología positiva busca que el individuo sea feliz con la vida que lleva, que se sienta motivado y capaz de enfrentar cualquier dificultad y alcanzar metas, (Gorjón-Gómez \&
Quintero-De-Moya, 2021). Con ayuda de la mediación que tiene una metodología para generar felicidad y paz, no sólo gestionar y transformar el conflicto (Gorjón, 2020).

Es importante señalar que al solucionar los conflictos con el proceso de mediación mejorará la cultura y el clima organizacional de la empresa, en la perspectiva social el no padecer de algún tipo de riesgo psicosocial contribuirá al mejoramiento de la calidad de vida del trabajador y la de su familia. Se evita con ello la angustia ese temor a sufrir una enfermedad laboral en los empleados que realizan sus tareas cotidianas dentro de la organización, buscando, además, fomentar la creatividad e innovación en sus actividades.

De la misma forma la prevención de los factores de riesgo psicosocial por medio de la mediación y al resolver conflictos que se dan dentro de la empresa evitaría pérdidas humanas ya que es lo más importante para la sociedad, lo que incrementara un mejoramiento de la salud pública ya que los trabajadores no están expuestos a agentes nocivos. Además, contribuirá a formar parte de un imperativo legal por parte del Estado evitando sanciones, citaciones por parte de los entes legales que regulan la seguridad y salud en el trabajo.

También se disminuirán las tasas de ausentismo, accidentabilidad y enfermedades laborales lo cual representa un beneficio para el empleador, empleado y la empresa al garantizarle condiciones de seguridad, salud y bienestar en el ambiente de trabajo, de esta forma con el establecimiento de normas 
y políticas de prevención de accidentes de trabajo y enfermedades laborales, se logrará que haya una disminución de los daños materiales y humanos, generando de esta manera una mejor imagen de la organización y un mayor incremento de la productividad ya que el personal estará motivado al trabajar fuera de riesgos, asimismo, no se debe olvidar que las competencias que logre el trabajador en materia de salud laboral serán de gran importancia para todos los que hacen parte de la organización.

\section{CONCLUSIONES}

Los factores de riesgo psicosocial no deben ser considerados como un tema superficial dentro de las organizaciones, la evidencia indica que los trabajadores se encuentran en condiciones de trabajo no adecuadas para realizar sus tareas, el trabajo trae consigo una serie de elementos que si bien ayudan al individuo a lograr su autorrealización también es verdad que puede generarle provocarle una serie de malestares físicos y psicológicos que pueden desencadenar un determinado tipo de enfermedad laboral.

La administración y la gerencia de toda empresa deben asumir su responsabilidad en buscar y poner en práctica las medidas necesarias que contribuyen a mantener $y$ mejorar los niveles de eficiencia en las operaciones, y brindar a sus trabajadores un ambiente laboral sano y seguro. Resolver conflictos dentro de la organización antes de que se produzca un riesgo psicosocial por medio del proceso de mediación es sin duda un gran paso en las empresas que quieran adoptar este mecanismo alternativo que busca forjar un mayor bienestar biopsicosocial en los individuos.

\section{TRABAJOS CITADOS}

Aguirre, E. (1994). Seguridad integral en las organizaciones actualización para ejecutivos. México, D.F.: Trillas.

Bonafé-Schmitt, J.-P. (2006). La médiation scolaire par les pairs. Spirale - Revue de Recherches en Éducation, (37) pp. 173-182. Recuperado en: https://spirale-edu-revue. fr/IMG/pdf/15_Bonaffe_Spirale_37.pdf

Charria, V., Sarsosa, K., \& Arenas, F. (2011). Factores de riesgo psicosocial laboral: métodos e instrumentos de evaluación. Revista Facultad Nacional de Salud Pública, 29 (4) pp. 380 - 391. Recuperado en: https://www. redalyc.org/articulo.oa?id=12021522004

Chiavenato, I. (2007). Administración de recursos humanos. México, D.F.: Mc Graw Hil.

Cortés Diaz, J. M. (2015). Seguridad e higiene del trabajo. Técnicas de Prevención de Riesgos Laborales. México, D.F.: Tébar Flores.

De Armas Herández, M. (2003). La mediación en la resolución de conflictos. Educar, (32) pp. 125 - 136. Recuperado en: https://raco.cat/index.php/Educar/article/ view/20783/20623

De la Poza, J. M. (1990). Seguridad e higiene profesional con las normas comunitarias europeas y norteamericanas. Madrid: Paraninfo.

Díaz Naranjo, D. L. (2011). Estrés laboral y sus factores de riesgo psicosocial. Revista CES Salud Pública, 2 (1) pp. 80 - 84.Recuperado en: https://dialnet.unirioja.es/servlet/articulo?codigo $=3677229$

Durand de San Juan, E. A \& Rodríguez-Burgos, K. E. (2015). Uso de la mediación en solución de conflictos en el mercado de arrendamiento pp. 133 - 156 en Hernández Paz, A. A., \& y Sánchez García, A. (Eds.) Solución de Conflictos en las Relaciones Comerciales Internacionales Mexico, D.F.: Rechtikal.

Garrido-Pinzón, J., Uribe-Rodríguez, A. F. \& Blanch, J. M.(2011). Riesgos Psicosociales desde la Perspectiva de 
la Calidad de Vida Laboral. Revista Acta Colombiana de Psicología, 14 (2) pp. 27 - 34. Recuperado en: https:// www.redalyc.org/articulo.oa?id=79822611003

Gil-Monte, P.R.(2012). Riesgos psicosociales en el trabajo y salud ocupacional. Revista Peruana de Medicina Experimental y Salud Pública, 29 (2) pp. 237 - 241. Recuperado en: http://www.scielo.org.pe/pdf/rins/v29n2/ a12v29n2.pdf

Gorjón Gómez, F. J. (2020). La mediación como política de bienestar. Revista Polífica, Globalidad y Ciudadanía, 6 (12) pp. 67 - 83. Recuperado en: https://revpoliticas. uanl.mx/index.php/RPGyC/article/view/147/205

---12016). Mediacion, ciencia social emergente. Comunitania. Revista Internacional de Trabajo Social y Ciencias Sociales, (12) pp. 9 - 25. Recuperado en: http://revistas.uned.es/index.php/comunitania/article/ view/18933/15825

Gorjón Gómez, F J., \& Pesqueira Leal, J. (2015). La ciencia de la mediación. México, D.F.: Tirant Lo Blanch.

Gorjón Gómez, F. J., \& Quintero De Moya, H. F.(2021). La justicia terapéutica como política de bienestar social. Revista Iberoamericana de Justicia Terapéutica (2). Recuperado en: https://ar.ijeditores.com/index.php?option=publicacion\&idpublicacion $=594$

Herzberg, F. (1987). How do you motivate employees? Harvard Busines Review, pp. 5 - 16. Recuperado en: https://kyleshulfermba530.weebly.com/ uploads/2/3/4/5/23454770/one_more_time_how_do_ you_motivate_employees.pdf

Martínez-Pérez, Y. B., Sauceda Villeda, B. J., \& Moreno Rodríguez, M. S. (2021). Los derechos procesales en grupos vulnerables desde la normativa nacional e internacional. Revista Política, Globalidad y Ciudadanía, 6 (12) pp. 225 - 245. Recuperado en: https://revpoliticas.uanl. $\mathrm{mx} /$ index.php/RPGyC/article/view/155/212

Martínez Pérez, Y. B., \& Gorjón Gómez G. J. (2018). Prócticas restaurativas en el marco jurídico mexican o con enfoque a una cultura de paz. Revista Pensamiento Americano, 11 (21) pp. 79 - 95. Recuperado en: https://publicaciones. americana.edu.co/index.php/pensamientoamericano/ article/view/153/171

Miranda-Medina, C.F., Martínez Pérez, Y. B., García Salvador, K., Hernández Martínez, A., Rodríguez Medrano, K., \& y Sosa Ruiz, M. (2019). Percepción sobre la violencia en las relaciones amorosas de los estudiantes de una Facultad de Derecho y Criminología de una Universidad del Norte de México. Erg @omnes Revista Jurídica, 11 (1) pp. 171 - 195. Recuperado en: https://revistas.curn.edu. co/index.php/ergaomnes/article/view/1504/1090

Moreno Jiménez, B. (2011). Factores y riesgos laborales psicosociales: conceptualización, historia y cambios actuales. Revista Medicina y Seguridad del Trabajo, 57 (1) pp. 4 - 19. Recuperado en: https://scielo.isciii.es/pdf/ mesetra/v57s1/especial.pdf

Murúa Chevesich, H. y Granda Ibarra, A. (1977). Manual de seguridad e higiene del trabajo. La Habana: Orbe.

Organización Internacional del Trabajo (OIT). (2020). Seguridad y salud en el trabajo. Recuperado en: https:// www.ilo.org/global/topics/safety-and-health-at-work/ lang--es/index.htm

Organización Panamericana de la Salud (OPS) y Organización Mundial de la Salud (OMS). (2003). Salud de los Trabajadores: Recursos - Preguntas Frecuentes. Recuperado en: https://www.paho.org/hq/index.php?option=com_content\&view=article\&id=1527: workers-health-resources\&ltemid $=1349 \&$ limitstart $=2 \&$ lang $=e s$

Polo-Vargas, J.D., Palacio Sañudo, J. E., De Castro Correa, A.M., Mebarak Chams, M. R., \& y Velilla Guardela, J. L. (2013). Riesgos psicosociales: la psicología organizacional positiva como propuesta de promoción, prevención e intervención. Revista Salud Uninorte, 29 (3) pp. 561 - 575. Recuperado en: https://rcientificas.uninorte. edu.co/index.php/salud/article/view/5813/3566

Raffo Lecca, E., Ráez Guevara, L., \& Cachay Boza, O. (2013). Riesgos psicosociales. Revista de Investigación Industrial Data, 16 (1) pp. 70-79. Recuperado en: https://revistasinvestigacion.unmsm.edu.pe/index.php/idata/article/view/6420/5642 
Rentería Valencia, J. A., Fernández Ospina, E., Tenjo, A. M., \& y Uribe Rodrìguez, A. F. (2009). Identificación de factores psicosociales de riesgo en una empresa de producción. Revista Diversitas Perspectivas en Psicología, 5 (1) pp. 161 - 175. Recuperado en: https://revistas.usantotomas.edu.co/index.php/diversitas/article/view/232/381

Rivas-Castillo, C., Rodríguez-Burgos, K., \& Miranda-Medina, C. (2020). La ciencia, tecnología e innovación en América Latina. Revista Cuaderno Jurídico y Políitico, 6 (16) pp. 6-17. Recuperado en: https://www.camjol.info/ index.php/cuadernojurypol/article/view/11105/12968

Ruiz, A. (1987). Salud ocupacional y productiva. México, D.F: Limusa.

Sauceda Villeda, B. J., \& Martínez Pérez, Y. B. (2018). Los MASC desde el Sistema para el Desarrollo Integral de la Familia en México. Revista de la Facultad de Derecho, (44) pp. 1 - 31. Recuperado en: http://eprints. uanl.mx/13565/1/607-Texto\%20del\%20art\%C3\%ADculo-2119-1-10-20180401.pdf

Vieco Gómez, G. F., \& y Abello Llanos, R. (2014). Factores psicosociales de origen laboral, estrés y morbilidad en el mundo. Revista Psicología desde el Caribe, 31 (2) pp. 354 -386. Recuperado en: https://www.redalyc.org/articulo.oa?id=21331836004

$-$

\section{Hamudy Quintero}

Psicólogo, Magister en Gerencia De Recursos Humano y Especialista en Seguridad y Salud en el Trabajo con 15 años de experiencia en el sector empresarial y 10 años en el sector Educativo; Maestría Gerencia de Recursos Humano, Universidad Experimental de las Fuerzas Armadas (UNEFA); Especialista en Seguridad y Salud en el Trabajo: Universidad Simón Bolívar; Experiencia en el sector educativo: Universidad Simón Bolívar, Universidad reformada, Servicio Nacional de Aprendizaje (SENA) y la Base Naval de Barranquilla. 
